\title{
Measurements and modelling of the residual mass upon impact of supercooled liquid drops
}

\author{
Mark Gloerfeld $^{1}$ (D) - Ilia V. Roisman ${ }^{1} \cdot$ Jeanette Hussong ${ }^{1} \cdot$ Cameron Tropea $^{1}$
}

Received: 9 June 2021 / Revised: 6 August 2021 / Accepted: 7 August 2021 / Published online: 20 September 2021

(C) The Author(s) 2021

\begin{abstract}
The mass of liquid remaining on a substrate following a drop impact is a crucial quantity for modelling of numerous phenomena, e.g. spray cooling, spray coating or aircraft icing. In the present study, a method to measure this residual mass after impact of liquid drops is introduced. This method is also applicable to supercooled drops, which may freeze upon impact on cold surfaces. Using the data obtained from extensive measurements in which the size, impact speed and temperature of the drops was varied, a modelling of the residual mass is formulated, following closely the theory of Riboux and Gordillo (Phys Rev Lett 113(2):024507, 2014. https://doi.org/10.1103/PhysRevLett.113.024507). A key adaptation of this model accounts for the deformation of drops immediately prior to impact. This modified theoretical model results in very good agreement with experiments, allowing prediction of residual mass for a given impact situation.
\end{abstract}

\section{Graphical abstract}

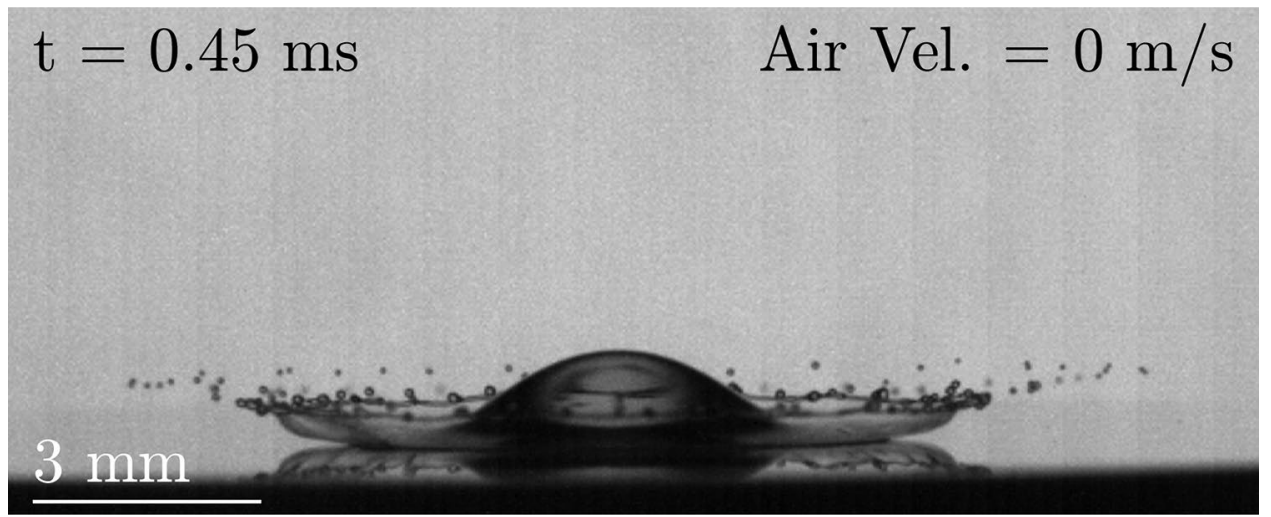

\section{Introduction}

Drop impact is a widely studied phenomenon in the laboratory with a myriad of applications in both the physical and life sciences. Although the hydrodynamics have been extremely well elucidated, experimentally, numerically and theoretically (Yarin 2006; Josserand and Thoroddsen 2016; Yarin et al. 2017), one quantity remains extremely elusive

Mark Gloerfeld

gloerfeld@sla.tu-darmstadt.de

1 Institute of Fluid Mechanics and Aerodynamics, Technische Universität Darmstadt, Alarich-Weiss-Strasse 10, 64287 Darmstadt, Germany for the case of a drop impact exceeding the splash threshold, namely the amount of residual liquid remaining on the surface, or alternatively, the amount of liquid splashed and re-emitted during the impact. In all cases, the residual mass on the surface is a quantity of great interest since it directly influences subsequent processes such as heat transfer (spray cooling (Breitenbach et al. 2018)), wetted area (spray coating (Andrade et al. 2013), encapsulation, leaf coverage (Papierowska et al. 2019)) and ice accretion (Szilder et al. 2002). Some work regarding the residual mass of a drop detaching from a fibre is available (Aziz et al. 2018; Jamali et al. 2021; Holweger et al. 2021); however, the involved physics likely differ significantly from the mass loss from 
drop splashing on plane substrates. One main difficulty in formulating models to describe the deposited mass in dependence of the material properties and boundary/initial conditions of the drop impact is the lack of reliable experimental data. The result is the existence of widely varying empirical correlations presently available in the literature (Moreira et al. 2010).

The mechanisms of splash in the case of drop impact onto a wetted wall are well known. An uprising liquid sheet bounded by a Taylor rim - a corona - appears and expands as a result of a propagation of a kinematic discontinuity along the wall film (Yarin and Weiss 1995; Roisman and Tropea 2002). This discontinuity is a result of interaction of the radially spreading lamella, generated by drop impact, and the outer unperturbed liquid film. For a single drop impact, the splashing threshold is determined by the impact parameters, liquid properties and the relative initial wall film thickness (Cossali et al. 1997). For different liquids in the drop and the wall film, the viscosity ratio is also an important factor influencing the splashing threshold (Kittel et al. 2018; Geppert et al. 2017).

One example of splash of a drop after an impact onto a wetted wall (Kittel 2019) is shown in Fig. 1. The formation of the secondary drops is caused by the transverse instability of the rim (Roisman 2010) formed at the free upper edge of the corona by capillary forces (Taylor 1959; Culick 1960). The size of the secondary drops in this case is comparable with the diameter of the corona rim cross section (Roisman et al. 2007).

The splashing on a dry solid substrate is completely different phenomena. The threshold is influenced not only by the impact parameters, but also by the substrate morphology, like roughness or porosity (Marengo et al. 2011; Roisman et al. 2015) and by the ambient pressure (Xu et al. 2005; Xu 2007; Stevens et al. 2014). More recently, the role of aerodynamic effects in the vicinity of the propagating contact

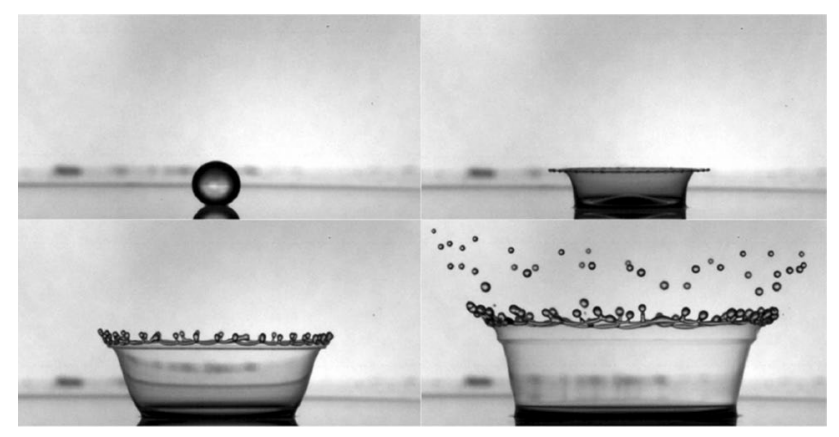

Fig. 1 Corona rim splash of a silicon oil drop impacting onto a thin liquid film. The drop initial diameter $D_{0}=2 \mathrm{~mm}$ and the impact velocity are $U_{0}=3 \mathrm{~m} / \mathrm{s}$. The oil kinematic viscosity is $5 \mathrm{~mm}^{2} / \mathrm{s}$. The initial film thickness is $h=250 \mu \mathrm{m}$. Images courtesy of Dr. Hannah Kittel, TU Darmstadt line in the formation of the corona after drop impact onto a perfectly smooth dry substrate has been analyzed in Riboux and Gordillo (2014, 2017). The models presented in these articles explain the formation of the corona and predict the splashing threshold and the main properties of the secondary drops (Riboux and Gordillo 2015). For drop impact onto soft, elastic substrates or coatings, also the material properties of the targets become significant factors of splashing (Coux et al. 2017; Alizadeh et al. 2013; Chen et al. 2016).

Several examples of splash on a dry substrate are shown in Fig. 2. It has been shown in Burzynski et al. (2020) that the splash after high-speed drop impact is caused not necessarily by the rim instability, as in Fig. 1, but by the Rayleigh-Taylor instability of the free liquid sheet. This unstable corona can be seen on the left series of images in Fig. 2. If the breakup length of the corona is smaller than the lamella diameter, the corona does not appear. The corresponding prompt splash is shown in the right series of images in Fig. 2.

If the phenomenon is not isothermal also thermodynamic effects, namely the effects associated with the phase change of the drop during impact and spreading, can significantly influence the hydrodynamic phenomena and lead to different splashing regimes. In the case of a liquid drop impacting onto a very hot substrate, these effects include mainly nucleate or film boiling (Liang and Mudawar 2017; Breitenbach et al. 2018). For drop impact onto a very cold substrate, the flow is influenced by near wall solidification, especially near the contact line (Herbaut et al. 2019; De Ruiter et al. 2017).

The freezing and solidification of a supercooled drop on a solid substrate occur in stages, the first being the fast expansion of a cloud of thin ice dendrites from the embryo ice crystal reaching a critical size (Libbrecht 2017). During their formation, the dendrites release heat into the surrounding bulk liquid until an equilibrium is reached, leaving
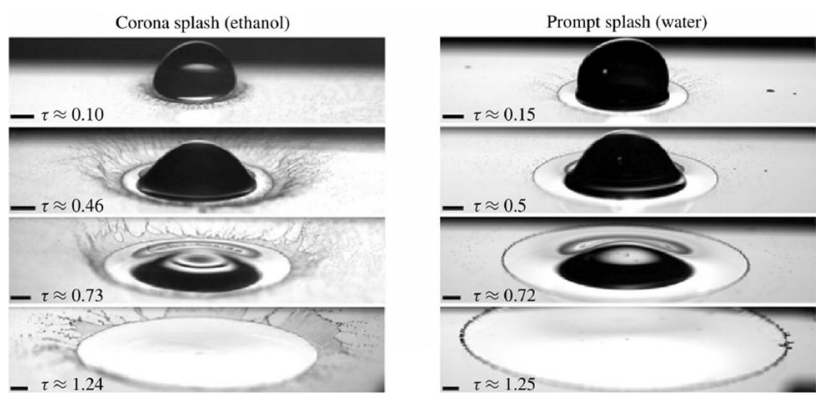

Fig. 2 Comparison of the phenomena of corona splash (left image of an ethanol drop impact with initial drop diameter $D_{0}=2.5 \mathrm{~mm}$ and impact velocity $U_{0}=13 \mathrm{~m} / \mathrm{s}$ ) and prompt splash (right image of a distilled water drop impact with $D_{0}=3.7 \mathrm{~mm}$ and $U_{0}=10 \mathrm{~m} / \mathrm{s}$ ) captured using a high-speed video system (from Burzynski et al. 2020). The dimensionless time after the impact instant is defined as $\tau=t U_{0} / D_{0}$. With permission from Cambridge University Press 
a mixture of ice dendrites and water at the melting temperature. Impact of a supercooled drops is accompanied by a freezing delay on dry substrates. The statistics of the freezing delay after drop impact onto metal targets has been evaluated in Schremb et al. (2017b). The instant of the drop freezing is determined by the processes associated with heterogeneous ice nucleation at the substrate. An overview of current studies on various icing phenomena accompanying drop impact, water spreading and receding can be found in a recent review paper (Roisman and Tropea 2020).

Splashing leads to the reduction of the residual liquid mass on the substrate in comparison with the initial mass. Principally, there can be two approaches to the mass of the deposited, residual part of the impacting drop; either the mass remaining on the surface after the impact is measured, or the mass of ejected/splashed liquid is measured. Both pose rather difficult experimental challenges. For the latter, some attempts have been made to measure secondary drops emitted from the splash, but as visible in Fig. 2, this is difficult, since the trajectory and density of drops can vary significantly depending on the type of splash encountered prompt splash or corona splash. Using a point measurement technique like phase Doppler for characterization of the secondary spray is not convenient since the mass flux measurement is not particularly reliable (Albrecht et al. 2003). The prospect of improving such a measurement using high-speed video cameras and image processing to detect and size all ejected secondary droplets has improved over the past years; however, the achievable depth of field while maintaining a large field of view and high spatial resolution, and the diffraction-limited resolution of small secondary droplets can result in significant uncertainties. Thus, also, the method based on the image processing is only applicable for certain specific situations in which these limiting factors can be marginalized.

The alternative approach is to measure the residual mass on the impact surface, and this is also the approach addressed in the present study. Also here, several factors limit the achievable accuracy. One difficulty is that splashed droplets or ejected ice fragments in the case of impacting ice crystals may not be ejected far from the impact point and simply fall back onto the surface, thus remaining indistinguishable from the main residual bulk. Two avenues have been followed in the past to avoid this problem. One is to investigate the impact of drops vertically upwards onto a surface, in which case any emitted mass will fall away from the surface. This of course imposes different boundary conditions on the problem. The second avenue is to use a small target, in which case the deposition of re-emitted mass is reduced, if not eliminated. Neither of these methods are ideal, but may be acceptable, depending on the application.

In the present study, a novel approach to determining residual mass on an impact target is introduced. This method has originally been developed for the impact of supercooled large drops in liquid and mixed phase, as well as for ice crystals exhibiting a fragmentation upon impact, where the residual mass may be in a solid (frozen) state. This particular application is related to ice accretion in the aviation industry, where the residual mass becomes an important quantity in the development of accretion models.

\section{Experimental setup}

In this study, the impact of supercooled large droplets (SLD) onto a small target is examined. The experiments are conducted in a wind tunnel mounted in a cooling chamber. The chamber (inside dimensions $2.2 \mathrm{~m} \times 1.8 \mathrm{~m} \times 2.4 \mathrm{~m}$ ) maintains ambient temperatures down to $T_{\mathrm{amb}}=-20^{\circ} \mathrm{C}$; thus determining the temperature of the airflow, the drops and the impact target. All temperatures range within $\pm 1.5^{\circ} \mathrm{C}$ of the chamber temperature $T_{\mathrm{amb}}$. Furthermore, the ambient air is fully saturated, thus avoiding any temperature variation due to drop evaporation.

\subsection{Icing wind tunnel and impact surface}

The wind tunnel is a vertical open return blower wind tunnel placed inside a cooling chamber, as depicted in Fig. 3. After passing the inlet section and nozzle, the flow enters a square test section with a side length of $140 \mathrm{~mm}$, before entering a diffusor leading to a radial fan. The flow exits the fan back into the cooling chamber. The velocity profile

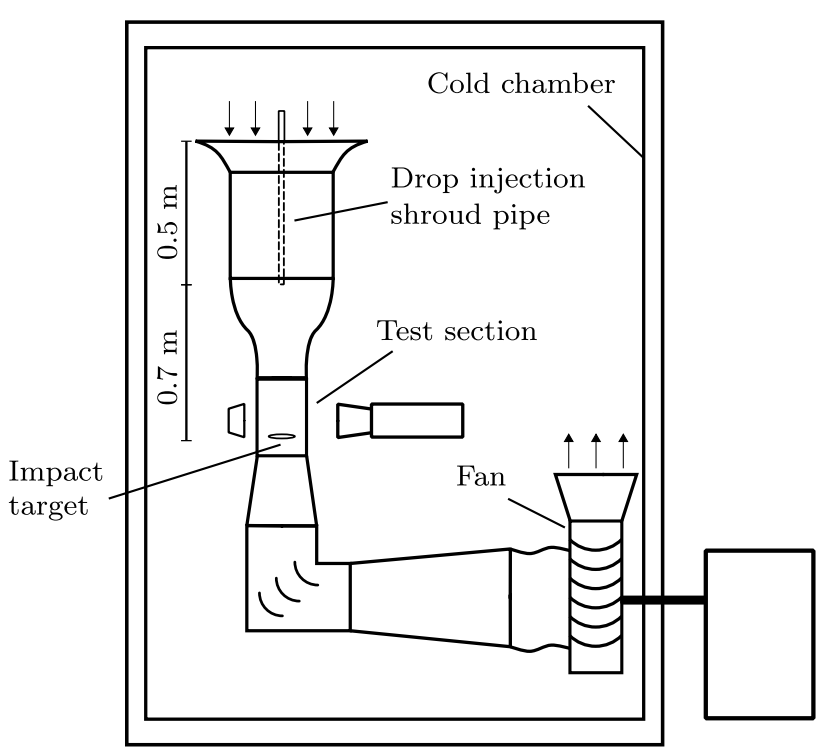

Fig. 3 Wind tunnel setup for investigating the impact of supercooled water drops. Some components in the sketch are not to scale 
across the test section is highly uniform, reaching velocities up to $U_{\text {air }}=40 \mathrm{~m} / \mathrm{s}$ with a turbulence intensity of Tu $=0.5 \%$.

Supercooled drops are generated inside the tunnels settling chamber using a syringe needle inside of the wind tunnel. They are protected from the airflow by a shroud pipe during their formation. After detaching from the syringe, the drops accelerate due to gravity until entering the air flow, where they are further accelerated by the flow. In the test section, the drops impact onto a flat aluminium target, placed horizontally in the wind tunnel. The impact is captured using a high-speed camera (Photron SA-X2) with background illumination.

In order to quantify the supercooling of the drops, the temperature is continuously measured by a thermocouple inside of the syringe needle, as introduced by Schremb et al. (2017a). Additionally, the temperatures of the surface and the air flowing through the test section are monitored during the experiments.

To examine the deposited liquid or ice on the surface after drop impact, some additional features have been incorporated into the target, as depicted in Fig. 4. A heating wire wraps around the cylindrical aluminium target in order to melt any residual ice remaining on the target. This is especially helpful in retroactively determining the volume/mass of the deposited fluid, since after heating the eventually frozen drop becomes a sessile, liquid drop. To examine the residual ice and/or sessile drop in more detail, the target can be rotated, during which images from numerous viewing angles are captured. This technique, employing multiple views, yields a high precision measurement, elaborated in more detail below.

\subsection{Drop volume estimation}

In order to estimate the deposited mass, the target is first heated to melt any ice which formed during or after the impact. When melted, the liquid recedes into a sessile drop, whose exact geometry is determined by the volume of the liquid and the substrate wettability. Several images of this sessile drop are then captured from different viewing angles by rotating the target. In this manner, any deviations from

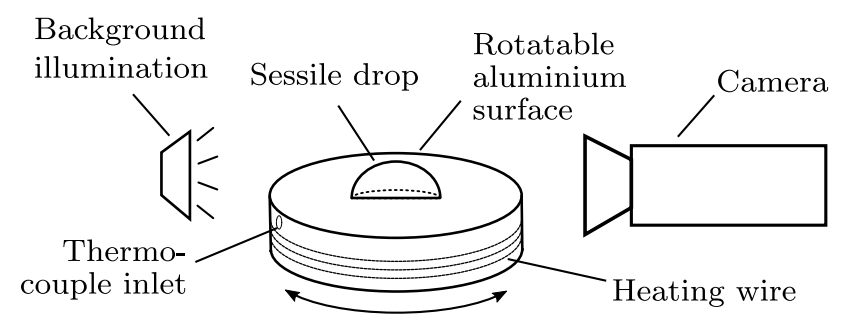

Fig. 4 Rotatable impact surface for the investigation of residual mass, enabling multiple viewing angles of a sessile drop axial symmetry or off-centring are accounted for, increasing the precision of the determined volume. These images are used for three-dimensional reconstruction of the drop shape.

The drop apex is used as a fix point to align the various contours obtained from each image. After arranging and scaling the contours into a single bulk object, they are used to create a point cloud of the drop. For this purpose, an elliptical curve is fitted to horizontal slices of the constructed object. Thus, the curvature of the drop surface is included in the reconstruction. Any deviation from an elliptic surface would occur most likely immediately adjacent to the surface, caused by pinning of the contact line. However, the undisturbed receding of the drop further reduces the probability of strong deviations from a non-elliptic shape. From the dot cloud, a triangulation of the enclosed volume is performed. The method is calibrated using metal spheres exhibiting a precision better than $10 \mu \mathrm{m}$ in diameter. In order to estimate their volume, images between $0^{\circ}$ and $180^{\circ}$ are captured with an angular increment of $30^{\circ}$. During this calibration, a reconstruction of the sphere volume with a maximum deviation of $0.2 \mu \mathrm{l}$ is achieved. Thus, the method enables a reconstruction of drop volumes with an uncertainty of $\pm 0.2 \mu$ l.

\section{Results and discussion}

In some cases, the drops investigated in this study impact at very high speeds, leading to significant deformation of the drop before impact. The consequences of this deformation on the impact hydrodynamics and ultimately on the residual mass are now introduced and discussed.

\subsection{Observations of the impact of non-spherical drops}

To illustrate the drop deformation, two drops immediately prior to impact are visualized in Fig. 5. The drop in the left photograph of this figure impacts approximately with its terminal velocity, i.e. without an airflow. The drop in the
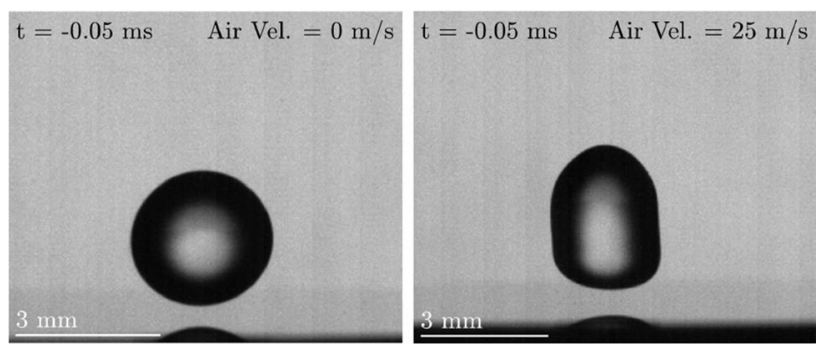

Fig. 5 Drop deformation before impact due to acceleration in the surrounding air flow. The left drop impacts without a co-flow. The right drop is accelerated by an air flow with $U_{\text {air }}=25 \mathrm{~m} / \mathrm{s}$. Both drops are of the same volume $\left(V_{0} \approx 14.1 \mu \mathrm{l}\right)$ 
right photograph is additionally accelerated in a co-flow with $U_{\text {air }}=25 \mathrm{~m} / \mathrm{s}$. Three features are immediately apparent. The drop is strongly deformed, exhibiting a flattening of the lower surface, a stronger curvature on the upper surface, and a slight asymmetry. These features become even more apparent in the picture sequences presented in Fig. 6, showing a drop with an impact velocity of $U_{0} \approx 9.5 \mathrm{~m} / \mathrm{s}$ in an air flow with $U_{\text {air }}=25 \mathrm{~m} / \mathrm{s}$. Here, we assume that the aerodynamic drag changes from a vertically downward force acting on the drop far from the wall to a vertically upward directed force as the drop is decelerated in proximity to the wall. Correspondingly, the stagnation end of the drop is flattened and the wake end exhibits suction and a bulging, the stagnation and wake ends changing as the drop approaches the substrate.

The light vertical asymmetry observed in these photographs and those in Fig. 9 can be attributed to the fact that the drop does not always impact exactly at the stagnation point of the gas co-flow on the target surface. Falling a distance of approx. $70 \mathrm{~cm}$ from entering the air flow to impact, it is not possible to control the impact position better than $\pm 0.5 \mathrm{~cm}$.

Associated with the deformed drop, the lower surface of the drop is flattened before impact. This shape change before impact presumably also affects the development of the expanding liquid lamella ejected from the drop impact. In order to quantify the effect of drop deformation before impact on this
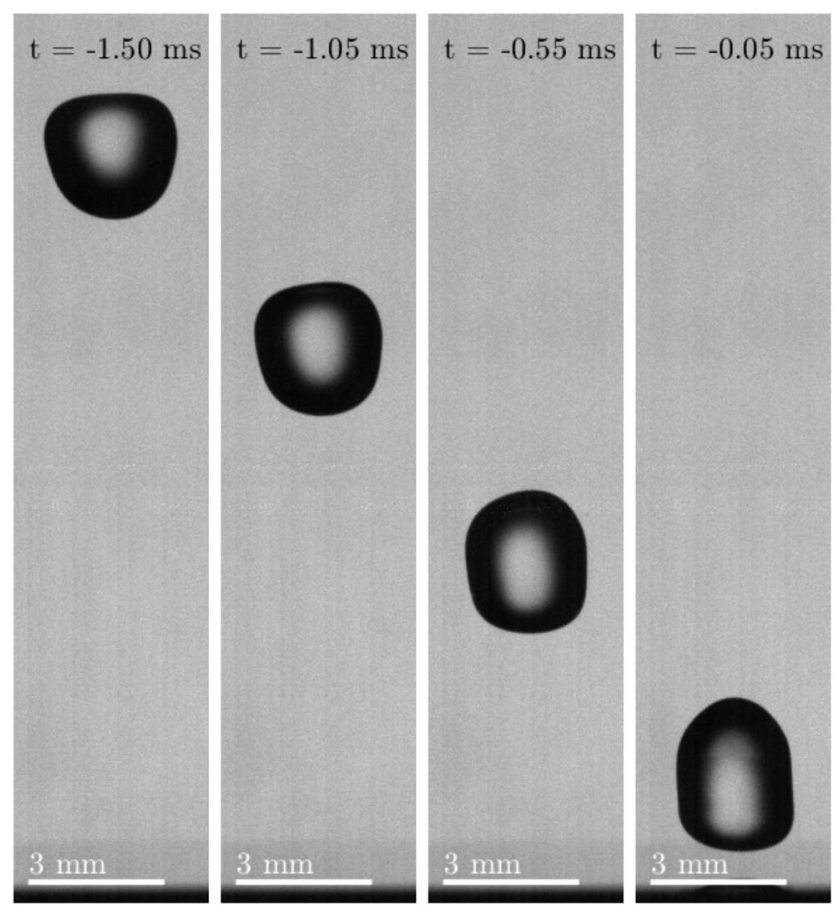

Fig. 6 Image sequence of a drop approaching the impact surface with $U_{0} \approx 9.5 \mathrm{~m} / \mathrm{s}$ in an air flow with $U_{\text {air }}=25 \mathrm{~m} / \mathrm{s}$ expanding liquid lamella, the radius of the drop as it spreads on the surface (wetted area) is evaluated.

Starting with the case of an impacting spherical drop as a reference, the radius of the wetted area during the initial kinematic stage of impact is given as $r \propto \sqrt{t}$ (Yarin 2006). It is obtained simply by truncating a sphere at a radius decreasing linearly with time and examining the radius of the cut cross section (assuming constant drop velocity upon impact). This leads to the dimensionless relation

$r^{+}\left(t^{+}\right)=b \sqrt{t^{+}}$

whereby $r^{+}=r / R_{0}$ is the dimensionless radius, $t^{+}=t U_{0} / R_{0}$ the dimensionless time and $b$ a dimensionless proportionality constant. $R_{0}$ is the radius of the spherical drop. Considering now the impact of a deformed drop, its wetted radius upon impact can be estimated using Eq. (1) with $b$ being of the order $\mathcal{O}(2)$. If now the radius of the lower drop surface differs significantly from the spherical value $R_{0}$, then the value of $b$ will also differ. Denoting the curvature (inverse radius) of the lower drop surface as $\kappa$ and by applying a least square fit to the wetting radius growth with time obtained from the evaluation of the high-speed recordings, $b$ can be determined for every impacting drop event. This experimental result is shown in Fig. 7, indicating that $b$ decreases for increasing values of the dimensionless curvature $\kappa R_{0}$, i.e. when the drop lower surface flattens before impact, the spreading of the drop on the surface after impact increases in velocity.

A first-order model for the quantity $b$ is proposed as

$b=\frac{b_{0}}{\sqrt{\kappa R_{0}}}$.

where $b_{0}$ corresponds to the proportionality constant of a spherical drop marked with a vertical dashed line in Fig. 7. A least square fit of Eq. (2) with $b_{0}=2.12$ is plotted in Fig. 7

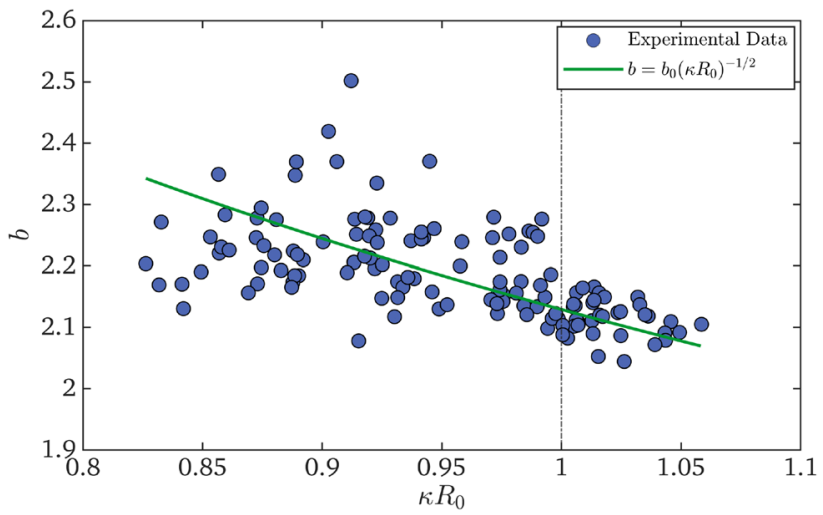

Fig. 7 Proportionality constant $b$ as a function of the dimensionless curvature $\kappa R_{0}$ of the impacting drops lower surface 
as a straight, solid line. The value for $b_{0}$ agrees well with Rioboo et al. (2002), who suggest a value of $b_{0}=2.05$ for spherical drops (at $\kappa R_{0}=1$ ). Note that the original value given in their work is obtained with a different scaling, which requires a conversion of the value with the factor $1 / \sqrt{2}$ in order to compare it to the findings of the present study.

Finally, inserting Eq. (2) into Eq. (1) leads to a relation for the wetted area $r(t)$ no longer dependent on $R_{0}$ but on $\kappa$; thus, supporting the hypothesis that the initial flow on the surface is governed by the curvature of the lower drop surface instead of the radius representative of its volume, $R_{0}$. The scatter of the data in Fig. 7 is likely influenced by the fact that the experimental values were obtained from a single projected side view of the impacting drop from the highspeed recordings. An observation of the spreading from the top or bottom perspective would presumably provide more consistent data of the average spreading velocity. However, Fig. 7 clearly reveals a dependency between $b$ and the local drop curvature.

An accurate evaluation of the kinematics of spreading, accounting for the local drop curvature, is necessary for an improved description of the conditions for the corona development and splash, and thus the fluid volume remaining on the surface. The parameters taken into account are now introduced following closely the theory presented by Riboux and Gordillo (2014).

\subsection{Conditions for the corona splash}

The supercooled water drops examined in this study exhibit a corona splash upon impact, which is not immediately selfevident. At temperatures around $20^{\circ} \mathrm{C}$ a drop impacting with comparable size and impact velocity would exhibit a prompt splash or deposition (Palacios et al. 2012). The change into the corona splash regime is attributed to the low temperature of the water drops. When water is supercooled, its viscosity increases significantly, for instance, the viscosity of water increases by a factor of 3.3 for a temperature change from $20^{\circ} \mathrm{C}$ to $-15^{\circ} \mathrm{C}$ (VDI and GVC 2006). According to Roisman et al. (2015), the threshold parameters for the prompt/ corona splash regimes are well-defined using a critical Ohnesorge number $\mathrm{Oh}_{\text {crit }}=\eta / \sqrt{\rho D_{0} \sigma}=0.0044$, whereby $\eta$ is the liquid dynamic viscosity, $\rho$ the liquid density, $D_{0}$ the diameter of the impacting drop and $\sigma$ the surface tension. A corona splash is predicted for values larger than $\mathrm{Oh}_{\text {crit }}$. Considering the change in fluid properties due to supercooling, this condition is fulfilled for all drops investigated in this study, as illustrated in Fig. 8. This figure shows the Weber number $\mathrm{We}=\rho U_{0}^{2} D_{0} / \sigma$ of all examined drop impacts plotted over the corresponding impact Reynolds number $\operatorname{Re}=\rho D_{0} U_{0} / \eta$, where $U_{0}$ is the impact velocity of the drop normal to the target. The critical Ohnesorge number is shown in this figure as

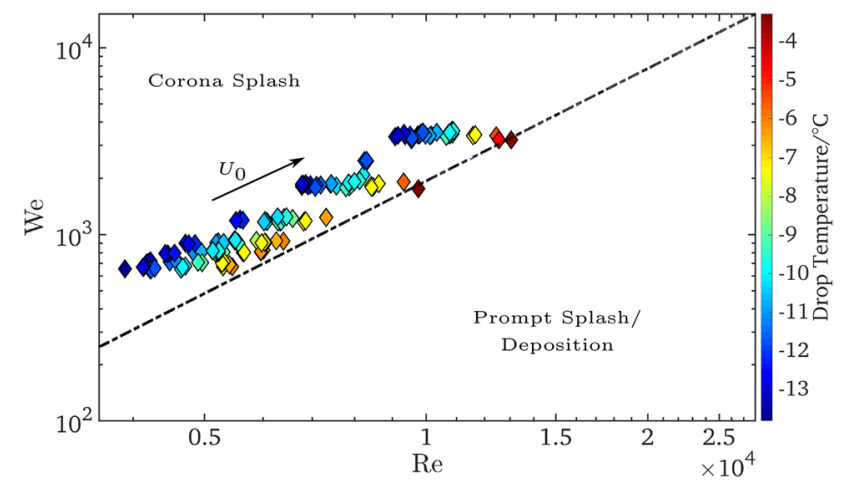

Fig. 8 We and Re of all investigated drop impacts. The marker colour indicates the drop temperature which is also accounted for in the properties used to calculate the dimensionless numbers. The dashed line marks the critical Ohnesorge number $\mathrm{Oh}_{\text {crit }}=0.0044$

a dashed line. The temperature of the examined drops ranges between $T_{0}=-3.3^{\circ} \mathrm{C}$ and $T_{0}=-13.8^{\circ} \mathrm{C}$.

The drop temperature, represented in Fig. 8 by the symbol colour, indicates that a change in temperature results in a significant change of the Reynolds number Re; however, the Weber number We is hardly affected, since the fluid properties contained in We $(\rho, \sigma)$ only change slightly. Since the Ohnesorge number is independent of the impact velocity, the transition to the corona splash regime is merely caused by the fluid properties and the drop diameter. As indicated by the arrow in Fig. 8, an increasing impact velocity entails a transition to higher We and Re, yet no transition to the other splashing regimes occurs. Considering a change of Oh due to drop size, a prompt splash/deposition of a supercooled drop $\left(T_{\text {drop }}<0{ }^{\circ} \mathrm{C}\right)$ is only possible for large droplets. In fact, according to this threshold, supercooled drops smaller than $D_{\text {drop }}=2200 \mu \mathrm{m}$ will always exhibit a corona splash on a smooth surface. Moreover, larger droplets will also exhibit a corona splash for a relatively small increase in supercooling.

Although all drops observed in the present study impact with a corona splash, the extent of the splash differs with temperature and air flow velocity, i.e. impact velocity. This change is observable qualitatively in Fig. 9, which shows the instant before the thin film of the corona breaks up for the highest and lowest temperatures and impact velocities, respectively. Comparing Fig. 9a, b, a slight influence of the temperature on the splash is noticeable. The crown of the corona of the colder drop extends farther away from the impact position just before breakup. We assume that the increased time until breakup and the ensuing increased extent of the splash originate from a more stable film during crown formation; the stabilization presumably being a consequence of the increased viscosity at lower temperatures.

A higher impact velocity will increase the extent of the corona distinctly. When comparing Fig. 9c, d, a change due to an increased impact velocity is observable. For the higher 


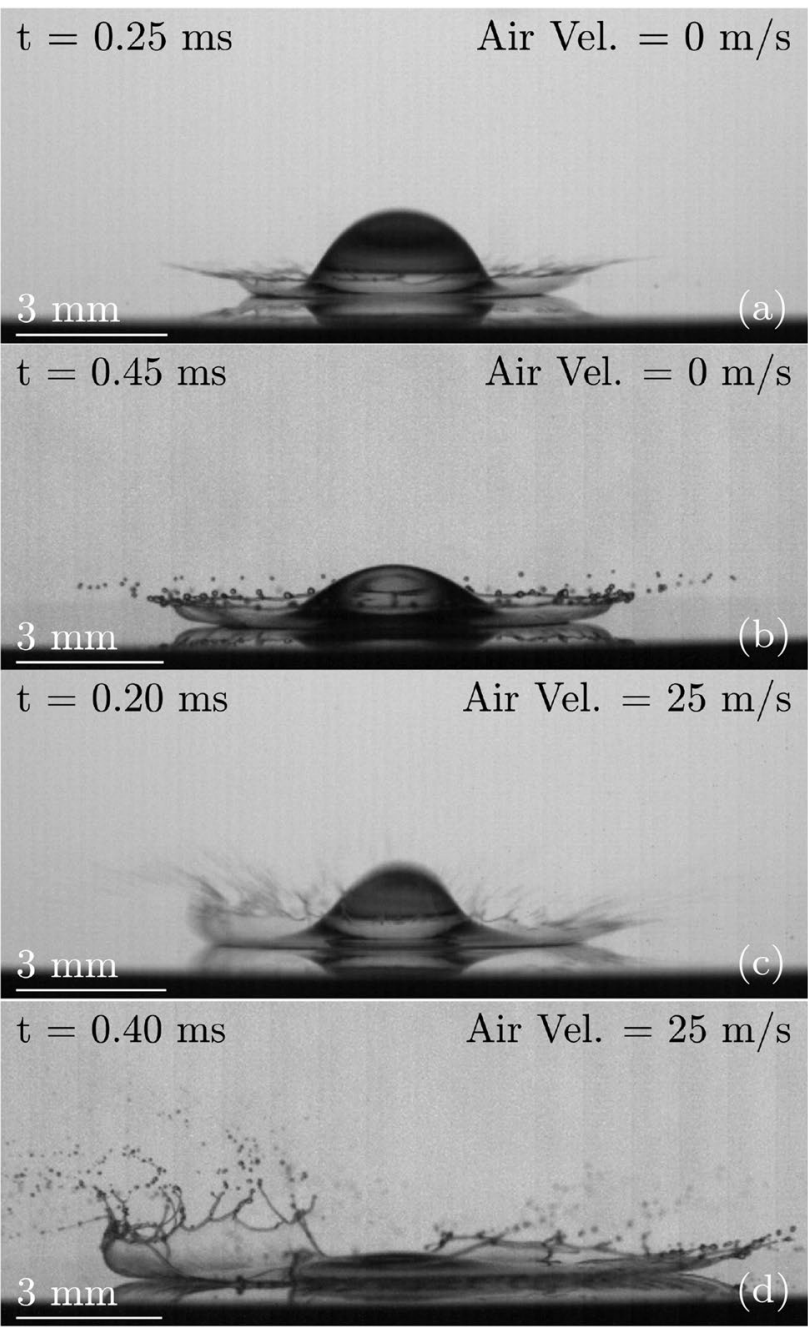

Fig. 9 Comparison of the corona extends in the incident before break up from four drops $\left(D_{\text {drop }}=3000 \mu \mathrm{m}\right)$ with different drop temperatures and impact velocities. a $U_{0} \approx 4.2 \mathrm{~m} / \mathrm{s}, T_{0} \approx-5^{\circ} \mathrm{C}$; b $U_{0} \approx 4.2 \mathrm{~m} / \mathrm{s}, \quad T_{0} \approx-13^{\circ} \mathrm{C} ; \quad$ c $U_{0} \approx 10.5 \mathrm{~m} / \mathrm{s}, \quad T_{0} \approx-5^{\circ} \mathrm{C} ; \quad$ d $U_{0} \approx 10.5 \mathrm{~m} / \mathrm{s}, T_{0} \approx-13^{\circ} \mathrm{C}$

velocity, the film of the corona spreads faster. An additional increase in supercooling enhances the spreading of the corona even more. Again, the uprising film is stable for a longer period of time, providing more time for its expansion. The asymmetries observed in the photographs of Fig. 9c, $\mathrm{d}$ can be attributed to drop deformation before impact and the wall tangential flow superimposed on the splashed drop.

According to the theory of Riboux and Gordillo (2014), the corona propagation during drop impact onto a dry smooth substrate is governed mainly by the inertia of the flow in the lamella, surface tension and aerodynamic effects acting on the propagation of the contact line. This propagation is described using the dimensionless parameter $\beta$, defined in their work as the ratio of the aerodynamic lift force acting on the spreading lamella to the surface tension

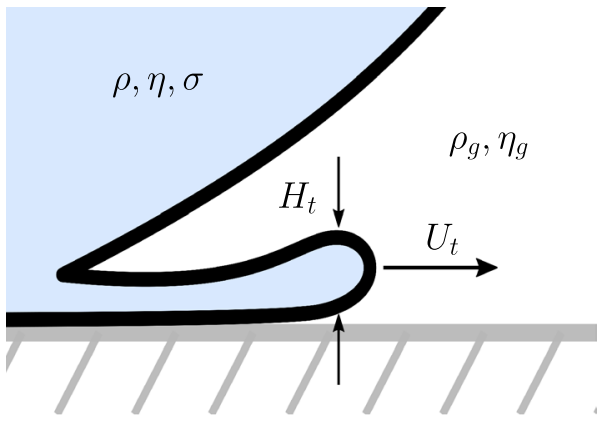

Fig. 10 Lamella height $H_{t}$ and velocity $U_{t}$ in moment of lift-off $t=t_{e}$ used to determine $\beta$ based on Riboux and Gordillo (2014)

force. A critical value $\beta^{\star} \simeq 0.14$ is given as the splashing threshold. Hence, a splash occurs, if the impact parameters satisfy the condition $\beta>\beta^{\star}$. It should be noted that the impact parameters in this study also satisfy this condition of the corona generation on a solid substrate, caused by the aerodynamic stresses in the fast-propagating contact line.

In order to calculate $\beta$, the instant $t_{e}$ at which the lamella starts to lift from the surface is required. The fluid and gas properties and the dimensions of the lamella considered in the model at $t=t_{e}$ are outlined in Fig. 10. To determine $t_{e}$, Riboux and Gordillo (2014) provide an implicit correlation with regard to the impact conditions as

$\frac{\sqrt{3}}{2} \operatorname{Re}_{R}^{-1} t_{e}^{-1 / 2}+\operatorname{Re}_{R}^{-2} \mathrm{Oh}_{R}^{-2}=1.21 t_{e}^{3 / 2}$.

Here, $\mathrm{Re}_{R}$ and $\mathrm{Oh}_{R}$ refer to the dimensionless Reynolds and Ohnesorge number, respectively. As indicated by the index $R$, they are defined using the initial drop radius $R_{0}$ as $\operatorname{Re}_{R}=\rho U_{0} R_{0} / \eta$ and $\mathrm{Oh}_{R}=\eta / \sqrt{R_{0} \rho \sigma}$. With knowledge of $t_{e}$, the lamella thickness $H_{t}$ and its velocity $U_{t}$ in the moment of lamella lift-off can be calculated according to

$H_{t}=R_{0} \sqrt{12} / \pi t_{e}^{3 / 2}$

and

$U_{t}=\sqrt{3} / 2 U_{0} t_{e}^{-1 / 2}$.

The parameter $\beta$ is obtained from a balance of the lamella lift force and the fluid surface tension as

$\beta=\sqrt{\frac{K_{l} \eta_{g} U_{t}+K_{u} \rho_{g} U_{t}^{2} H_{t}}{2 \sigma}}$

with $\eta_{g}$ and $\rho_{g}$ being the surrounding gas dynamic viscosity and density, respectively. The parameters $K_{l}$ and $K_{u}$ result from contributions to the lift force from lubrication and suction. While $K_{u}$ is approximately constant $\left(K_{u} \simeq 0.3\right), K_{l}$ 
depends on the mean free path of the gas molecules $\lambda$ and $H_{t}$. It is estimated using the relation

$K_{l} \simeq-2\left[\ln \left(19.2 \lambda / H_{t}\right)-\ln \left(1+19.2 \lambda / H_{t}\right)\right]$.

Note, that Eqs. (4) and (7) are taken from the erratum published to Riboux and Gordillo (2014). Assume now that any deformation of the drop prior to impact also causes a change in wetting radius propagation and therefore in the spreading velocity of the lamella. Hence, the expression for the tangential velocity of the lifting sheet $U_{t}$ must be adjusted with the correction term from Eq. (2), i.e. $U_{t, c}=U_{t} / \sqrt{\kappa R_{0}}$. Additionally, the radius $R_{0}$ is replaced by the inverse of the curvature $\kappa$ in $\mathrm{Re}_{R}, \mathrm{Oh}_{R}$ and in the term for the sheet thickness $H_{t}$ i.e. $H_{t, c}=H_{t} /\left(\kappa R_{0}\right)$.

\subsection{Correlation for the residual drop volume}

The mass ejected from the drop during a corona splash is strongly connected to the development of the liquid sheet lifting from the surface. As indicated in Fig. 9, the extent and shape of this sheet until it collapses varies with impact velocity as well as with liquid viscosity. Whereas an increase in viscosity is expected to result in a more stable film, an increasing impact velocity presumably lifts the sheet earlier. Both effects provide more time for fluid to enter the liquid sheet before emerging instabilities lead to its breakup. In order to analyse the residual volume on the surface as a function on impact velocity and liquid viscosity, the capillary number $\mathrm{Ca}=\mu U_{0} / \sigma$ is introduced. Figure 11 shows the residual volume fraction $V_{\text {res }} / V_{0}$ plotted in relation to $\mathrm{Ca}$. It is apparent that for higher $\mathrm{Ca}$ lower residual volumes occur. However, the large scatter for higher $\mathrm{Ca}$ leaves great uncertainty in a clear decreasing trend. Considering the exclusion of drop size and shape in this empirical view, a more physical approach is thought to be more elucidating,

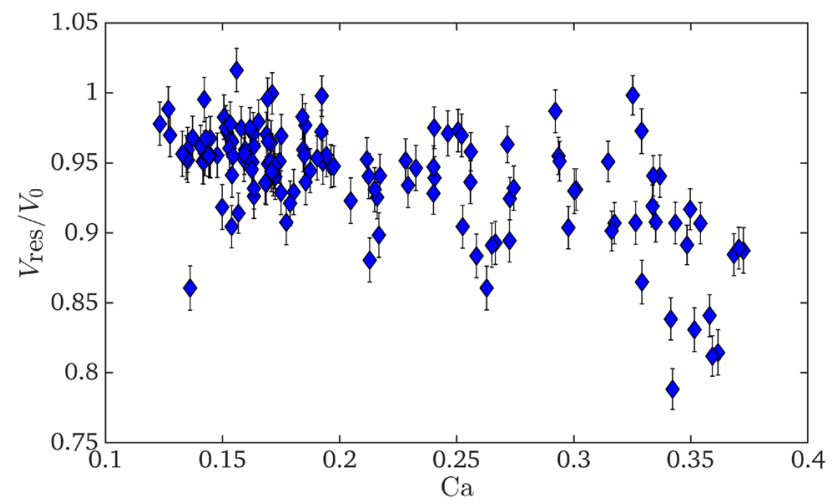

Fig. 11 Residual volume fraction of supercooled drops in relation to $\mathrm{Ca}$ which leads to the above-mentioned model from Riboux and Gordillo (2014).

The characteristics affecting the residual volume are combined in the parameter $\beta>\beta^{\star}$, as successfully used in Burzynski et al. (2020). Moreover, in contrast to the approach using $\mathrm{Ca}$, the model provides the possibility to include changes conditional on the drop shape, as introduced earlier. In the present study, this hypothesis is followed by examining the measured residual volume as a function of $\beta$ (Fig. 12) for Ohnesorge numbers $\mathrm{Oh}>0.055$, corresponding to a well-developed corona, far from the threshold for prompt splash. The data for $V_{\text {res }} / V_{0}$ correlate well with the values of the $\beta$ parameter, supporting the model of Riboux and Gordillo (2014). The data are supplemented with values obtained by Burzynski et al. (2020), which agree well with the decreasing trend of the residual volume for increasing $\beta$.

However, the scatter in the data from this study and data of Burzynski et al. (2020) represented in Fig. 12 by the error bars, indicate that some influencing parameters are still unaccounted for. The parameter $\beta$ only characterises the impact during the very first stages of splashing. One can expect that at later stages of the lamella spreading further factors enter the problem, for instance the presence of Rayleigh-Taylor instabilities (Burzynski et al. 2020). Moreover, the air flow in the present experiments will also have an influence, which can only be quantified by performing additional experiments in which the drop impact point is systematically varied with respect to the air flow stagnation point on the target surface. Nevertheless, the results presented in Fig. 12, revealing a strong correlation between the factor $\beta$ and the residual volume on the surface, is a valuable result, for which there are very few alternatives presently available in the literature.

From Eqs. (3) to (6), it is apparent that the functional dependence of parameter $\beta$ on material properties and impact parameters is complex. Nevertheless, it is interesting to investigate whether the residual volume exhibits an additional

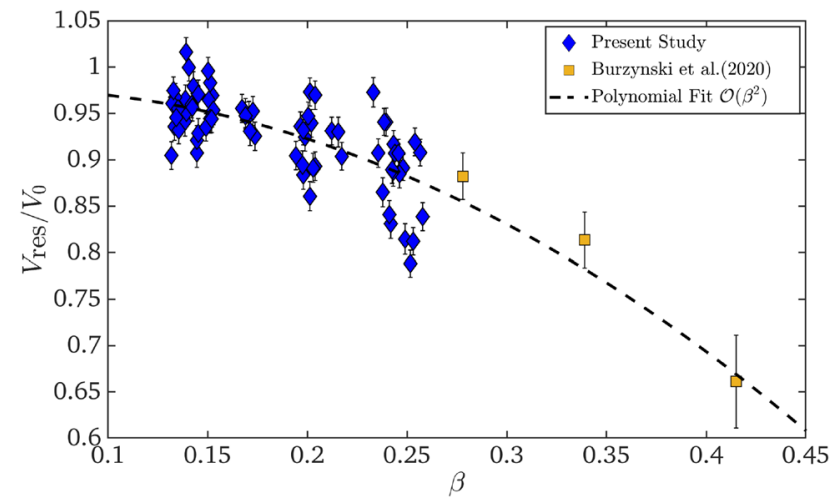

Fig. 12 Residual volume fraction of supercooled drops with $\mathrm{Oh}>0.055$ in relation to splashing parameter $\beta$ 


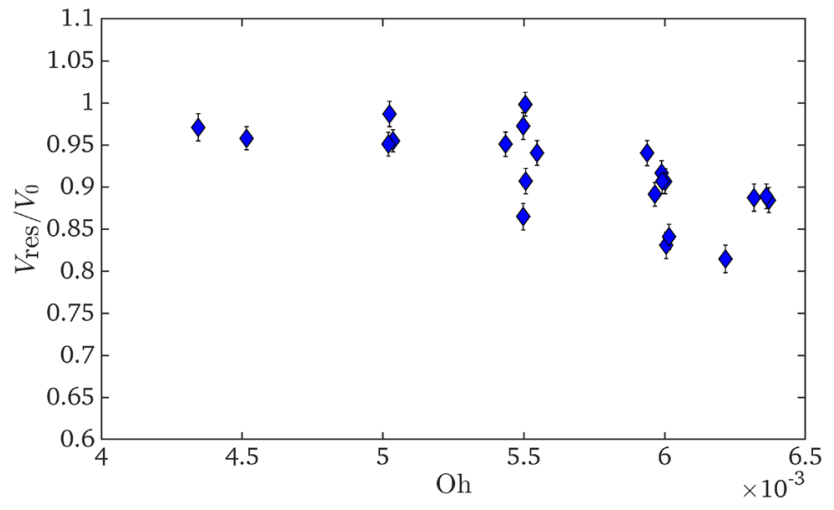

Fig. 13 Residual volume fraction of supercooled drops with the impact parameters corresponding to $\beta \approx 0.24$ as a function of $\mathrm{Oh}$

systematic dependence on Re, We or Oh. Many combinations have been investigated, and one representative example of this dependence of residual volume on $O h$ is shown in Fig. 13 for values of $\beta \approx 0.24$. As expected, the residual volume decreases with increasing Ohnesorge number; yet, this trend is not universal since the influence of $\beta$ visible in Fig. 12 is not included. In several of their studies, Riboux and Gordillo point out that under certain conditions, the parameter $\beta$ can be represented by a combination of Re, We or Oh (Riboux and Gordillo 2014; García-Geijo et al. 2021). However, regarding the physics determining the residual volume, some influence of the forces represented by the Ohnesorge number is still unaccounted for in the parameter $\beta$. Thus, no parameter combination is able to account for all influences responsible for the residing volume.

Nevertheless, in an attempt to illuminate to a greater degree the dependence of residual volume on impact parameters, the correlation between residual volume and the parameter $\beta$ evident in Fig. 12 has been used to compute some dimensioned relations. To do this, first a polynomial fit to the data in Fig. 12 was determined which is shown in the figure as a dashed line. This fit was then used to compute the residual mass, given changes in various parameters entering the quantity $\beta$. The results of this computation are shown in Fig. 14 for variations of the impact velocity, impact drop diameter and dynamic viscosity of the drop liquid. In all cases, an increase in these quantities leads to less residual volume. Note that a larger value of drop diameter as well as an increase in impact velocity has the same effect as a lower curvature of the impacting surface of the drop. Thus, a more flattened drop upon impact will result in a lower value of residual volume.
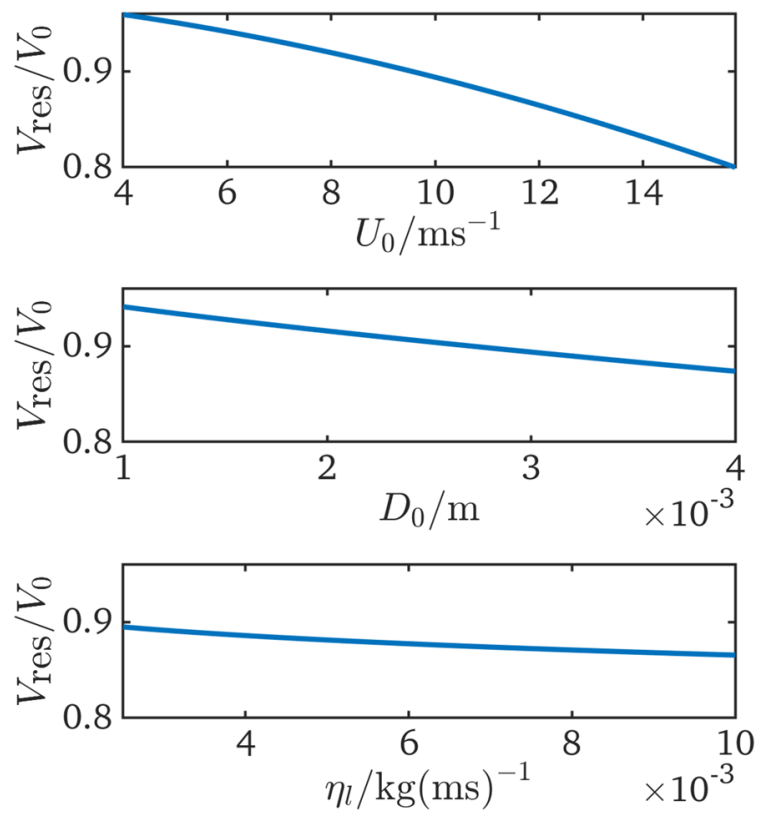

Fig. 14 Residual volume change according to variation of a single parameter based on the polynomial fit in Fig. 12. Computations performed for $U_{0}=10 \mathrm{~ms}^{-1}, D_{0}=3 \mathrm{~mm}$ and $\eta_{l}=0.0026 \mathrm{~kg}(\mathrm{~ms})^{-1}$ $\left(T_{0}=-10^{\circ} \mathrm{C}\right)$

\section{Summary and conclusions}

In this study, a method for determining the volume of liquid residing on the substrate after a drop impact at high Reynolds and Weber number has been introduced. This method is based on background illumination imaging from many viewing angles. By using a heated substrate, the technique is also applicable when using supercooled large drops, the residual volume of which may freeze on the target substrate after impact. The accuracy and repeatability of this method have been shown to be very high, exhibiting a volume estimation with a precision of $\pm 0.2 \mu \mathrm{l}$.

Having access to the residual mass after drop impact, experiments were then performed on supercooled drops impacting onto a dry surface at subzero temperatures. Both the temperature and the impact velocity were systematically varied. The effect of the temperature accounted for by the temperature dependence of the material properties of the liquid. High-speed recordings of the impact confirmed the occurrence of a corona splash for all investigated drops, leading to a reduction of the amount of fluid remaining on the surface (residual volume).

An existing model (Riboux and Gordillo 2014) was then used to predict the residual volume as a function of the material properties and dynamic impact parameters. This model had to be modified to account for drop deformation prior to impact. Such drop deformation is likely unavoidable when performing experiments with high drop impact 
velocities and a superimposed co-flow. Very satisfactory results between predictions and experiments were achieved by introducing the local curvature of the drop lower surface just prior to impact into the model.

A strong correlation between the splashed volume (or residual mass) and the $\beta$ parameter defined in Riboux and Gordillo (2014) was found. An additional dependence on the Ohnesorge number independent of $\beta$ revealed that effects unaccounted for in $\beta$ are likely to have an influence as well. At relatively high values of the Ohnesorge number, the residual volume can be described sufficiently well using only the parameter $\beta$.

Future investigations will examine more closely the residual volume for smaller values of the Ohnesorge number near the threshold of the prompt splash and the influence of the air flow during impact.

Supplementary Information Additional material including data of all conducted experiments and example videos of drop impacts observed in this study are found in Gloerfeld et al. (2021).

Acknowledgements The authors wish to acknowledge financial support of the Deutsche Forschungsgemeinschaft through the project SFBTRR 75. They also thank D. Burzynski for furnishing his data and for enlightening discussions about the comparability to our findings.

Author Contributions MG contributed to investigation, data curation, methodology, writing — original draft; IVR was involved in data curation, conceptualization, methodology, writing —original draft, writing-review and editing; JH contributed to onceptualization, supervision, writing - review and editing; CT contributed to conceptualization, supervision, writing — original draft, writing — review and editing.

Funding Open Access funding enabled and organized by Projekt DEAL.

Open Access This article is licensed under a Creative Commons Attribution 4.0 International License, which permits use, sharing, adaptation, distribution and reproduction in any medium or format, as long as you give appropriate credit to the original author(s) and the source, provide a link to the Creative Commons licence, and indicate if changes were made. The images or other third party material in this article are included in the article's Creative Commons licence, unless indicated otherwise in a credit line to the material. If material is not included in the article's Creative Commons licence and your intended use is not permitted by statutory regulation or exceeds the permitted use, you will need to obtain permission directly from the copyright holder. To view a copy of this licence, visit http://creativecommons.org/licenses/by/4.0/.

\section{References}

Albrecht HE, Damaschke N, Borys M, Tropea C (2003) Laser doppler and phase doppler measurement techniques. Springer, Berlin

Alizadeh A, Bahadur V, Shang W, Zhu Y, Buckley D, Dhinojwala A, Sohal M (2013) Influence of substrate elasticity on droplet impact dynamics. Langmuir 29(14):4520-4524. https://doi.org/10.1021/ la304767t
Andrade R, Skurtys O, Osorio F (2013) Drop impact behavior on food using spray coating: fundamentals and applications. Food Res Int 54(1):397-405. https://doi.org/10.1016/j.foodres.2013. 07.042

Aziz H, Farhan NM, Vahedi Tafreshi H (2018) Effects of fiber wettability and size on droplet detachment residue. Exp Fluids 59(7):2606. https://doi.org/10.1007/s00348-018-2579-z

Breitenbach J, Roisman IV, Tropea C (2018) From drop impact physics to spray cooling models: a critical review. Exp Fluids 59(3):1-21. https://doi.org/10.1007/s00348-018-2514-3

Burzynski DA, Roisman IV, Bansmer SE (2020) On the splashing of high-speed drops impacting a dry surface. J Fluid Mech 892:A2. https://doi.org/10.1017/jfm.2020.168

Chen L, Bonaccurso E, Deng P, Zhang H (2016) Droplet impact on soft viscoelastic surfaces. Phys Rev E 94(6):063117. https://doi. org/10.1088/0960-1317/14/6/006

Cossali GE, Coghe A, Marengo M (1997) The impact of a single drop on a wetted solid surface. Exp Fluids 22(6):463-472. https://doi.org/10.1007/s003480050073

Coux M, Clanet C, Quéré D (2017) Soft, elastic, water-repellent materials. Appl Phys Lett 110(25):251605. https://doi.org/10. $1063 / 1.4985011$

Culick FE (1960) Comments on a ruptured soap film. J Appl Phys 31(6):1128-1129. https://doi.org/10.1063/1.1735765

De Ruiter R, Colinet P, Brunet P, Snoeijer JH, Gelderblom H (2017) Contact line arrest in solidifying spreading drops. Phys Rev Fluids 2(4):043602. https://doi.org/10.1103/PhysRevFluids.2. 043602

García-Geijo P, Quintero ES, Riboux G, Gordillo JM (2021) Spreading and splashing of drops impacting rough substrates. J Fluid Mech 917:A50. https://doi.org/10.1017/jfm.2021.313

Geppert A, Terzis A, Lamanna G, Marengo M, Weigand B (2017) A benchmark study for the crown-type splashing dynamics of one-and two-component droplet wall-film interactions. Exp Fluids 58(12):172. https://doi.org/10.1007/s00348-017-2447-2

Gloerfeld M, Roisman I, Hussong J, Tropea C (2021) Supplementary material to the measurements of the residual mass upon impact of supercooled liquid drops. TUdatalib. https://doi.org/10.48328/ tudatalib-553.2

Herbaut R, Brunet P, Limat L, Royon L (2019) Liquid spreading on cold surfaces: solidification-induced stick-slip dynamics. Phys Rev Fluids 4(3):033603. https://doi.org/10.1103/PhysRevFlu ids. 4.033603

Holweger HJ, Jamali M, Tafreshi HV (2021) Centrifugal detachment of compound droplets from fibers. Langmuir ACS J Surf Colloids 37(2):928-938. https://doi.org/10.1021/acs.langmuir.0c03317

Jamali M, Mehta KS, Holweger H, Amrei MM, Tafreshi HV (2021) Controlling detachment residue via magnetic repulsion force. Appl Phys Lett 118(19):191601. https://doi.org/10.1063/5.00521 41

Josserand C, Thoroddsen ST (2016) Drop impact on a solid surface. Annu Rev Fluid Mech 48:365-391. https://doi.org/10.1146/annur ev-fluid-122414-034401

Kittel HM (2019) Drop impact onto a wall wetted by a thin film of another liquid. Ph.D. thesis, Technische Universität Darmstadt, Darmstadt, Germany

Kittel HM, Roisman IV, Tropea C (2018) Splash of a drop impacting onto a solid substrate wetted by a thin film of another liquid. Phys Rev Fluids 3(7):073601. https://doi.org/10.1103/PhysRevFluids.3. 073601

Liang G, Mudawar I (2017) Review of drop impact on heated walls. Int J Heat Mass Transf 106:103-126. https://doi.org/10.1016/j.ijhea tmasstransfer.2016.10.031

Libbrecht KG (2017) Physical dynamics of ice crystal growth. Annu Rev Mater Res 47:271-295. https://doi.org/10.1146/annur ev-matsci-070616-124135 
Marengo M, Antonini C, Roisman IV, Tropea C (2011) Drop collisions with simple and complex surfaces. Curr Opin Colloid Interface Sci 16(4):292-302. https://doi.org/10.1016/j.cocis.2011.06.009

Moreira A, Moita AS, Panão MR (2010) Advances and challenges in explaining fuel spray impingement: how much of single droplet impact research is useful? Prog Energy Combust Sci 36(5):554580. https://doi.org/10.1016/j.pecs.2010.01.002

Palacios J, Hernández J, Gómez P, Zanzi C, López J (2012) Experimental study of splashing patterns and the splashing/deposition threshold in drop impacts onto dry smooth solid surfaces. Exp Therm Fluid Sci 44:571-582. https://doi.org/10.1016/j.expthermfl usci.2012.08.020

Papierowska E, Mazur R, Stańczyk T, Beczek M, Szewińska J, Sochan A, Ryzak M, Szatyłowicz J, Bieganowski A (2019) Influence of leaf surface wettability on the drop splash phenomenon. Agric For Meteorol 279(2):107762. https://doi.org/10.1016/j.agrformet. 2019.107762

Riboux G, Gordillo JM (2014) Experiments of drops impacting a smooth solid surface: a model of the critical impact speed for drop splashing. Phys Rev Lett 113(2):024507. https://doi.org/10. 1103/PhysRevLett.113.024507

Riboux G, Gordillo JM (2015) The diameters and velocities of the droplets ejected after splashing. J Fluid Mech 772:630-648. https://doi.org/10.1017/jfm.2015.223

Riboux G, Gordillo JM (2017) Boundary-layer effects in droplet splashing. Phys Rev E 96(1):013105. https://doi.org/10.1103/PhysRevE. 96.013105

Rioboo R, Marengo M, Tropea C (2002) Time evolution of liquid drop impact onto solid, dry surfaces. Exp Fluids 33(1):112-124. https:// doi.org/10.1007/s00348-002-0431-x

Roisman IV (2010) On the instability of a free viscous rim. J Fluid Mech 661:206-228. https://doi.org/10.1017/S0022112010002910

Roisman IV, Tropea C (2002) Impact of a drop onto a wetted wall: description of crown formation and propagation. J Fluid Mech. https://doi.org/10.1017/S0022112002002434

Roisman IV, Tropea C (2020) Wetting and icing of surfaces. Curr Opin Colloid Interface Sci 53:101400. https://doi.org/10.1016/j.cocis. 2020.101400

Roisman IV, Gambaryan-Roisman T, Kyriopoulos O, Stephan P, Tropea C (2007) Breakup and atomization of a stretching crown. Phys Rev E 76(2):026302. https://doi.org/10.1103/PhysRevE.76. 026302

Roisman IV, Lembach A, Tropea C (2015) Drop splashing induced by target roughness and porosity: the size plays no role. Adv Colloid
Interface Sci 222:615-621. https://doi.org/10.1016/j.cis.2015.02. 004

Schremb M, Roisman IV, Tropea C (2017a) Normal impact of supercooled water drops onto a smooth ice surface: experiments and modelling. J Fluid Mech 835:1087-1107. https://doi.org/10.1017/ jfm.2017.797

Schremb M, Roisman IV, Tropea C (2017b) Transient effects in ice nucleation of a water drop impacting onto a cold substrate. Phys Rev E 95(2-1):022805. https://doi.org/10.1103/PhysRevE.95. 022805

Stevens CS, Latka A, Nagel SR (2014) Comparison of splashing in high-and low-viscosity liquids. Phys Rev E 89(6):063006. https:// doi.org/10.1103/PhysRevE.89.063006

Szilder K, Lozowski EP, Reuter G (2002) A study of ice accretion shape on cables under freezing rain conditions. J Offshore Mech Arct 124:162-168

Taylor GI (1959) The dynamics of thin sheets of fluid II. Waves on fluid sheets. Proc R Soc A Math Phys 253(1274):296-312. https://doi. org/10.1098/rspa.1959.0195

VDI, GVC (2006) VDI-Wärmeatlas. Springer, Berlin

$\mathrm{Xu} \mathrm{L}$ (2007) Liquid drop splashing on smooth, rough, and textured surfaces. Phys Rev E 75(5):056316. https://doi.org/10.1103/PhysR evE.75.056316

Xu L, Zhang WW, Nagel SR (2005) Drop splashing on a dry smooth surface. Phys Rev Lett 94(18):184505. https://doi.org/10.1103/ PhysRevLett.94.184505

Yarin AL (2006) Drop impact dynamics: splashing, spreading, receding, bouncing.... Annu Rev Fluid Mech 38(1):159-192. https:// doi.org/10.1146/annurev.fluid.38.050304.092144

Yarin AL, Weiss DA (1995) Impact of drops on solid surfaces: selfsimilar capillary waves, and splashing as a new type of kinematic discontinuity. J Fluid Mech 283(-1):141. https://doi.org/10.1017/ S0022112095002266

Yarin AL, Roisman IV, Tropea C (2017) Collision phenomena in liquids and solids. Cambridge University Press, Cambridge. https:// doi.org/10.1017/9781316556580

Publisher's Note Springer Nature remains neutral with regard to jurisdictional claims in published maps and institutional affiliations. 